\title{
Postmortem biochemical investigations in hypothermia fatalities
}

\author{
Cristian Palmiere $\cdot$ Patrice Mangin
}

Received: 28 February 2012 / Accepted: 26 June 2012 / Published online: 7 July 2012

(C) Springer-Verlag 2012

\begin{abstract}
Despite the progress made during the past several decades in forensic pathology, the possibilities for the postmortem diagnosis of hypothermia remains relatively limited. Aside from histology and immunohistochemistry, numerous authors have investigated the postmortem biochemistry of hypothermia fatalities. Several biochemical markers (e.g., glucose, electrolytes, hormones, ketone bodies, and neurotransmitters) and various biological samples (e.g., blood, urine, heart, liver, skeletal muscle as well as pericardial and cerebrospinal fluids) have been proposed as potentially useful markers to improve the insufficient diagnostic efficacy of macroscopic and microscopic findings. The aim of this article is to review the medicolegal literature covering the postmortem biochemical investigations that are associated with hypothermia cases as well as report our own research results on this topic where possible.
\end{abstract}

Keywords Hypothermia · Forensic pathology · Postmortem diagnosis $\cdot$ Postmortem biochemistry

\section{Introduction}

Hypothermia is defined as a condition in which the core body temperature is below $35^{\circ} \mathrm{C}\left(95{ }^{\circ} \mathrm{F}\right)$ and is the consequence of situations in which the body's loss of heat exceeds its ability to generate heat [1]. In the past, injury and death

C. Palmiere $(\bowtie) \cdot$ P. Mangin

University Centre of Legal Medicine,

Rue du Bugnon 21,

1011 Lausanne, Switzerland

e-mail: cristian.palmiere@chuv.ch

C. Palmiere $\cdot$ P. Mangin

University Centre of Legal Medicine,

Rue Michel-Servet 1,

1211 Genève 4, Switzerland from hypothermia were thought to occur almost exclusively among people with cold exposure in extreme, outdoor climates. However, following medical reports published in the 1950s and 1960s, it became evident that hypothermia can also occur in temperate climates and indoors, especially early or late in life as well as in those with preexisting pathologies (e.g., organic brain diseases) or precipitating factors during exposure (e.g., acute alcoholism) [2-4].

Hypothermia can be subclassified in different ways, the simplest of which is to divide fatalities into those occurring on land (dry) and those occurring in water (wet or immersion hypothermia). In cases of immersion hypothermia, the individuals tend to succumb to the effects of the cold water more rapidly than they do on land. Indeed, water aspiration may determine or contribute to the fatal outcome, with the cause of death being drowning rather than hypothermia. Cases of dry hypothermia tend to be more complex to investigate $[1,5]$.

Although postmortem examination is important, its role is not to confirm hypothermia, but to rule out natural diseases or violence as incapacitating or contributing factors leading to the hypothermic state [5]. This makes the postmortem diagnosis of hypothermia relatively arduous in forensic death investigations [6].

Macroscopic features that are suggestive of hypothermia during autopsy include reddish-brown discoloration of the skin over the elbows and knees (frost erythema), bright red lividity, acute gastric erosions (Wischnewski's spots), pancreatic hemorrhages, synovial membrane hemorrhages, bloody discoloration of synovial fluid, signs of acute pancreatitis, and hemorrhages into the large muscles of the body, especially the iliopsoas muscle [1, 7-27]. However, only two findings (Wischnewski's spots and frost erythema) have been indicated as the most "typical" morphological signs of death due to hypothermia [7].

In the literature of fatal hypothermia cases, several histological findings have been described such as the fatty 
degeneration of the renal tubular epithelium (subnuclear vacuolization of renal tubular epithelial cells or the Armanni-Ebstein phenomenon) [1,28-30]; pancreatic cell necrosis with leukocyte infiltration; vacuolization of anterior pituitary gland cells, hepatocytes, pancreatic adenocytes, and adrenal and renal cells; hypoxic cardiac changes; fatty changes of cardiac myocytes, hepatocytes, and renal tubular cells [28-34]. The fatty degeneration of the renal tubular epithelium has been indicated as the most reliable histological finding used to diagnose hypothermia [1, 28]; other histological features that were described in literature have been rejected in more recent studies [35].

A decrease in the adrenocorticotropic hormone (ACTH) and thyroid-stimulating hormone (TSH) immunopositivity rate in the anterior pituitary gland in hypothermia fatalities was described by Ishikawa et al. [36, 37]. They postulated that in a cold environment, TSH and ACTH production initially increased to generate heat and was subsequently suppressed by the metabolic disorders of abnormal lipid metabolism in advanced hypothermia. Ishikawa et al. [38] also described reduced immunopositivity for noradrenaline and dopamine in the adrenal medulla of hypothermia cases, which was positively correlated with noradrenaline levels in cerebrospinal fluid. According to the authors, these findings suggested a terminal hypofunction of the sympathetic/adrenomedullary system involving the adrenal medulla in fatal hypothermia. In the same study, the authors observed that the hypothalamus presented different findings involving neuronal dopamine immunopositivity, which led to the conclusion that the hypothalamus and adrenal medulla can be characterized by specific immunohistochemical features of systemic stress responses in these cases.

Yoshida et al. [39] described a low hypothalamus neuronal chromogranin A immunopositivity in hypothermia cases. Quan et al. [40] observed low ubiquitin immunoreactivity in the midbrain periaqueductal gray matter in fatal hypothermia. Ishikawa et al. [41] found high myoglobin immunopositivity in the renal tubular epithelium cells and high ubiquitin immunopositivity in the distal and collecting tubules. According to Ishikawa et al., these findings suggested that secondary skeletal muscle damage occurs due to hypoxia during the survival period and that renal tubular ubiquitin system activation was due to myoglobin uptake following myoglobinemia. Lastly, Preuss et al. [7] studied the expression of heat shock protein 70 in the kidneys of hypothermia fatalities and found an increased expression of this protein in renal tubular epithelial cells and glomerular podocytes. Similar findings were reported by Kita [42] in the cerebral cortex.

The aim of this article is to review the medicolegal literature pertaining to the postmortem biochemical investigations of hypothermia fatalities. Some of these markers may be measured in blood, urine, pericardial and cerebrospinal fluids and can be used in situations in which fatal hypothermia is suspected in order to improve the insufficient diagnostic efficacy of macroscopic and microscopic findings. Additionally, given that postmortem biochemical analyses are currently performed in our medicolegal center, we have reported the conclusions of our own research where possible.

\section{Postmortem biochemical investigations}

\section{Electrolytes}

The investigations by Mant [43, 44] are among the first published studies concerning the autopsy diagnosis of accidental hypothermia. The author analyzed vitreous magnesium concentration in a series of hypothermia fatalities as well as a control group and concluded that it was not possible to correlate the magnesium levels with the duration of the hypothermic state or the macroscopic findings.

In a study performed on 385 medicolegal autopsy cases, Li et al. [45] analyzed postmortem calcium and magnesium levels in pericardial fluid. Compared with the other studied groups, the hypothermia group had higher calcium concentrations and lower magnesium levels and $\mathrm{Mg} / \mathrm{Ca}$ ratios.

Jacubeniene et al. [46, 47] collected heart, liver, and skeletal muscle samples from two series of hypothermia cases and analyzed the calcium and sodium levels to investigate the possibility that hypothermia could induce electrolyte disturbances. Their results showed no significant differences in the calcium levels of any of the analyzed tissues whereas sodium concentration was significantly greater in the skeletal muscle tissues of the hypothermia cases.

\section{Catecholamines}

A great contribution to the knowledge of biochemical changes occurring during hypothermia was revealed in the investigations carried out by Hirvonen et al. [48-52]. In a study performed in 1976 concerning 22 fatal cases of accidental or suicidal hypothermia [48], the authors found that histamine and serotonin concentrations in urine tended to be lower in cases of hypothermia than in control cases. However, the differences between the hypothermia and control cases could also have been due to the general dilution of urine due to cold diuresis. The authors therefore concluded that histamine and serotonin assays could not be regarded as reliable tests for hypothermia deaths. Conversely, measurements of urinary catecholamine levels appeared to be more promising, with higher average concentrations in the hypothermia cases than in the control cases. Even though these differences may be influenced by urinary dilution induced by cold diuresis, Hirvonen et al. recommended this analysis to confirm a reaction of the organism to hypothermic stress. The test for urinary glucose was positive in some 
hypothermia cases, which may have indicated that hyperglycemia occurred in the early phase of cold exposure, without excluding increased glucose levels due to a preexisting diabetes mellitus. The conclusions of this study were confirmed by a subsequent investigation [49] performed on 24 hypothermia deaths, in which Hirvonen and Huttunen found that both urinary adrenaline and noradrenaline were significantly more elevated in hypothermia cases than in violent and natural deaths. This study also demonstrated that adrenaline levels increased proportionally more than noradrenaline. Hirvonen et al. [50] also observed increases in adrenaline, noradrenaline, and free fatty acid concentrations in the plasma of patients undergoing cardiac operations in which hypothermia and bypass were used; the adrenaline levels increased proportionally more than noradrenaline. Hanhela et al. [51] studied the effects of hypovolemia, hypothermia, and their combination on the hormonal and metabolic responses of rabbits. They found that both hypovolemia and hypothermia activated the sympathicoadrenal system, with more prominent increases in blood adrenaline levels than noradrenaline in cases of hypothermia. These cases also tended to display increases in free fatty acid and blood corticosterone levels. An increase in blood glucose levels was also observed, likely caused by the enhanced secretion of adrenaline and corticosterone.

Sadler and Pounder [53] reported three cases of fatal hypothermia that included markedly elevated catecholamine levels when related either to urinary creatinine or to predicted daily catecholamine excretion. Thus, the authors emphasized the importance of relating the excretion of catecholamines to concomitant creatinine excretion in interpreting catecholamine levels. They also concluded that increased urinary catecholamine levels provided evidence of prolonged agonal stress in hypothermia. However, the adrenaline-to-noradrenaline ratio, proposed by Hirvonen and Huttunen [52] as a diagnostic marker for hypothermia fatalities, did not appear to add further diagnostic elements.

Zhu et al. [54] investigated catecholamine levels (adrenaline, noradrenaline, and dopamine) in 542 autopsy cases in postmortem serum from different sampling sites (i.e., the left and right heart chambers, subclavian and external iliac vein). The hypothermia cases had lower cardiac and peripheral blood adrenaline, noradrenaline, and dopamine. According to the authors, the low postmortem serum catecholamine levels (and small right-left cardiac differences) were characteristic of fatal hypothermia, whereas previous reports indicated that postmortem serum and urinary catecholamine levels increased due to stress from cold exposure. Despite the greatly elevated catecholamine concentrations compared to the clinical reference intervals, the authors concluded that these findings were suggestive of relatively less cardiovascular stress and/or milder deterioration of the sympathetic nerve terminals during agony in the death process due to cold exposure.
In a successive study pertaining to the immunohistochemistry of catecholamines in the hypothalamic-pituitary-adrenal system, Ishikawa [38] observed a positive correlation between noradrenaline levels in cerebrospinal fluid and noradrenaline immunopositivity in the adrenal medulla in hypothermia cases.

Pakanen et al. [6] analyzed urine catecholamine levels in 358 autopsy cases, including 145 cases with hypothermia as the main cause of death. They found that adrenaline was a better marker of hypothermia death than noradrenaline, as already demonstrated by Hirvonen and Huttunen [49]. The adrenaline-to-noradrenaline ratio was proposed as the best indicator of cold exposure to eliminate the significance of any possible variation in the postmortem urine concentrations. According to the authors, the ratio would be less sensitive than the adrenaline concentration yet more specific.

\section{Vitreous glucose}

Bray et al. [55] performed biochemical investigations on 13 airplane crash victims who had remained immersed in nearfreezing water for several days and found that the glucose concentration in the vitreous humor of the plane crash victims was significantly higher than the values obtained in a control group. These results suggested that the rapid chilling of the eye caused by cold water immersion at the time of death could inhibit postmortem glycolysis by inactivating glycolytic enzymes.

Bray [56] also investigated vitreous glucose levels in 133 autopsy cases in which death occurred outdoors. The author found that mean glucose levels were higher in the deaths caused by cold exposure than in other deaths occurring in the cold. However, individual cases could not be distinguished on the basis of biochemical investigations.

Coe [57] investigated vitreous sodium, potassium, chloride, urea nitrogen, and glucose levels in a group of 26 hypothermia fatalities, which were compared with two postmortem control groups (a group of 100 nondiabetic subjects who died in situations in which the environmental temperatures had not produced or contributed to hypothermia and a group of 27 acute traumatic deaths that occurred outside during the winter months) and with a group of 40 nondiabetic individuals admitted to the hospital for hypothermia. The author found a significant mean elevation of vitreous glucose levels in the hypothermia group. This result suggested that hyperglycemia was a possible result of the elevations of serum catecholamines in response to stress. In addition, a substantial period of time had elapsed between cold exposure and death, thereby allowing a corresponding elevation of the vitreous glucose to occur. Coe concluded that increases in the vitreous glucose levels of nondiabetic individuals could support the evidence of hypothermia. Nevertheless, he emphasized that 
not all hypothermia cases displayed increased vitreous glucose levels, which suggested that cold exposure did not invariably lead to hyperglycemia.

In our medicolegal center, we observed 22 hypothermia cases between 2008 and 2011 (12 cases with no ethanol in the blood and 10 with ethanol in the blood). The vitreous glucose concentration was determined in all cases, with the results varying between 0.1 and $4.7 \mathrm{mmol} / \mathrm{l}$. Only six cases had vitreous glucose values greater than $3.4 \mathrm{mmol} / \mathrm{l}$. Conversely, most cases had vitreous glucose concentrations not exceeding $1 \mathrm{mmol} / \mathrm{l}$ and were comparable to values displayed by other causes of death. These results indicate that cold exposure does not invariably produce a hyperglycemic state, which concurs with the conclusions by Coe that the increase in blood glucose concentrations that occurs during the first phases of hypothermia is not consistently found in vitreous after death due to postmortem glycolysis.

\section{Markers of renal function}

Zhu et al. [58-60] analyzed urea nitrogen, creatinine, and uric acid levels in pericardial fluid and postmortem serum from different sampling sites (right heart, left heart, subclavian vein, and external iliac vein). In an initial study [58] performed on 395 medicolegal autopsy cases, the authors found an elevation of urea nitrogen $(>30 \mathrm{mg} / \mathrm{dl})$ in cases of fatal hypothermia, especially in the postmortem serum obtained from the heart blood. According to the authors, dehydration or hemoconcentration and elevated protein catabolism could be responsible for this urea nitrogen increase in hypothermia fatalities. In a subsequent study [59] performed on 409 medicolegal autopsy cases, the authors examined postmortem pericardial fluid for urea nitrogen, creatinine, and uric acid. Hypothermia cases tended to display mild to moderate elevations in urea nitrogen, creatinine, and uric acid levels. The authors emphasized that due to the nature of the pericardial fluid itself, in which the turnover rate is slower than in blood, the elevated pericardial urea nitrogen, creatinine, and uric acid concentrations could suggest the existence of persistent metabolic deteriorations before death. In a third study [60] performed on 556 medicolegal autopsy cases, the authors compared the differences between the pericardial fluid and postmortem serum levels of urea nitrogen, creatinine, and uric acid. Postmortem serum was obtained from different sampling sites (right heart, left heart, and external iliac vein). For hypothermia cases, the authors observed similar distributions in postmortem serum and pericardial fluid levels for urea nitrogen and uric acid, which were both increased, suggesting a prolonged death process involving protein catabolism. The authors concluded that since significant changes in pericardial fluid levels became apparent hours later than in serum, elevated urea nitrogen, creatinine, and uric acid levels in pericardial fluid could suggest several hours of prolonged survival.

\section{Ketone bodies}

Teresiński et al. [61-63] investigated ketone body blood levels as a biochemical marker of hypothermia and the usefulness of its determining to support the diagnosis of death by hypothermia. The authors assumed that the hyperketonemia that occurred during hypothermia was the result of the combined effect of hypoglycemia and the enhanced release of counter-regulatory hormones (glucagon, adrenaline, noradrenaline, cortisol, growth hormone), which intensify fat catabolism. In a study involving 16 cases of hypothermia [61], the authors observed an inverse relationship between blood ketone levels and the severity of insobriety due to the direct antiketonemic action of ethanol, which modifies cellular redox imbalance and prevents ketone production in the liver. Though increased blood ketone levels can be considered a diagnostic test of death by hypothermia, the authors emphasized that normal blood ketone concentrations would not exclude the diagnosis of hypothermia in cases that displayed ethanol in the blood. Increased ketone levels in urine and vitreous can provide useful information to support the diagnosis of death by hypothermia. However, the authors highlighted that urine ketone values require cautious interpretation due to the possible lack of ketonuria in conditions of coexisting ketonemia and renal failure of shock etiology or in cases of decreased ketonemia and persisting ketonuria related to urine retention. Lastly, the authors concluded that the severity of ketosis cannot be simply evaluated based on vitreous ketones since the equilibrium between blood and vitreous is established over time, and rapid increases of blood ketones are not immediately reflected by parallel increases of vitreous ketone levels.

The results of our own experiences [64] concur with the conclusions of Teresiński et al. and confirmed the usefulness of the systematic determination of 3-beta-hydroxybutyrate, acetone, and isopropyl alcohol levels in several biological substrates (e.g., blood, urine, and vitreous humor) in cases of suspected hypothermia. We observed several hypothermia fatalities that displayed an inverse relationship between ketone body and ethanol levels in various biological samples (e.g., blood and vitreous humor). Additionally, the determination of ketone body levels in blood, urine, and vitreous humor can provide further information to characterize the metabolic profile accompanying hypothermia fatalities and quantify the biochemical disturbances that may have led to death.

Markers of cardiac function

Zhu et al. [65-68] and Wang et al. [69] evaluated the usefulness of atrial natriuretic peptide (ANP), brain natriuretic peptide (BNP), cardiac troponin $\mathrm{T}(\mathrm{cTnT})$, creatine kinase $\mathrm{MB}$ (CK-MB), cardiac troponin I (cTnI), and myoglobin (Mb) in 
blood, pericardial fluid, and cerebrospinal fluids in medicolegal autopsy cases. In a study [65] performed on 405 forensic autopsy cases, the authors tested cTnT levels in postmortem serum from different sampling sites (right heart, left heart, and external iliac vein) and pericardial fluid. They observed that in hypothermia cases, cTnT remained at levels lower than the cutoff values. According to the authors, these findings suggested milder terminal myocardial hypoxia and milder postmortem cTnT elevation due to the rapid cooling of the body before and/or after death. Moreover, the authors emphasized that cTnT determination could be useful in differentiating acute myocardial infarction from hypothermia fatalities. The latter displayed only diffuse interstitial edema and patchy myocardial eosinophilic changes, which did not appear to significantly contribute to the elevation of the cTnT levels. In a study performed on 263 forensic autopsy cases, Zhu et al. [67] analyzed pericardial levels of ANP, BNP, and cTnT and found that ANP and BNP values were significantly higher in hypothermia cases. The authors emphasized that the hypothermia cases had biochemical results that were similar to those found in cases of chronic congestive heart disease. Since a high postmortem pericardial BNP level and BNP/ANP ratio may indicate the severity and duration of cardiac insufficiency before death, the authors concluded that these results emphasized the contribution of cardiac failure in the death process, even in the absence of massive myocardial damage. Wang et al. [69] investigated CK-MB, cTnI, and Mb levels in pericardial and cerebrospinal fluids and compared them to levels in postmortem serum from the right heart blood of 295 forensic autopsy cases. The hypothermia fatalities had lower CK-MB levels in cerebrospinal fluid and right cardiac blood as well as lower cTnI and $\mathrm{Mb}$ levels in all substrates. According to the authors, cold exposure may minimize damage to the viscera, thereby suggesting that limited damage would occur to the myocardium and skeletal muscles during the death process. Moreover, a low-temperature environment prevents postmortem decomposition. Conversely, a mild elevation in CK-MB pericardial fluid levels, as observed in this study, could be caused by prolonged hypoxia.

\section{Pituitary hormones}

Ishikawa et al. [36, 37, 70] investigated ACTH, TSH, and growth hormone $(\mathrm{GH})$ levels in postmortem serum from right heart blood and cerebrospinal fluid with a focus on fatal hypothermia. In an initial study performed on 162 forensic autopsies [36], the authors observed that the hypothermia cases had ACTH serum levels that were similar to the clinical reference value (even higher in some cases). Conversely, ACTH concentrations in cerebrospinal fluid (CSF) and the CSF/serum ratio were significantly lower when compared with the other studied groups, which typically displayed much higher CSF levels than serum levels.
According to the authors, these findings were consistent with the hypothesis of cold exposure-induced activation of the hypothalamic-pituitary-adrenal axis. The authors postulated that in a cold environment, ACTH production could initially increase to generate heat but then be suppressed due to the metabolic disorders involving abnormal lipid metabolism in advanced hypothermia. They concluded that the low ACTH levels in cerebrospinal fluid (i.e., similar to serum levels) suggested an exhausted pituitary function due to prolonged exposure to the cold. In a subsequent study performed on 120 forensic autopsies [37], hypothermia fatalities had lower postmortem serum and cerebrospinal fluid TSH levels than the other studied groups. The authors interpreted these results as a confirmation of the hypothesis of a dysfunctional hypothalamic-adenohypophyseal system due to cold exposure and exhausted pituitary function in the hypothermia cases. In a cold environment, TSH production, as with ACTH production, could initially increase to generate heat, only to be thereafter suppressed by the metabolic disorders involving abnormal lipid metabolism as cooling progressed. In a third study performed on 116 forensic autopsies [70], the authors observed that the hypothermia cases displayed elevated postmortem serum GH levels when compared with clinical reference ranges, possibly indicating a positive correlation with the stress reaction rather than thermal stress.

\section{Isopropyl alcohol}

Isopropyl alcohol (IPA) can be detected in situations other than IPA exposure, which are characterized by ketosis and an elevated NADH/NAD + ratio, such as hypothermia. Teresiński et al. [63] observed increased blood IPA levels in hypothermia cases, which were likely related to ketosis and the transformation of acetone into isopropyl alcohol.

Our own research [71] confirmed that IPA may be considered a marker of ketoacidosis and a product of acetone metabolism in clinical conditions that present ketonemia. In a study performed on 400 forensic autopsy cases including hypothermia, diabetic ketoacidosis, and alcoholic ketoacidosis fatalities, we observed that the highest IPA and ketone body concentrations were found in the blood, urine, and vitreous humor in diabetic ketoacidosis cases. The hypothermia fatalities had detectable IPA values in all analyzed substrates. However, the concentrations were less significant than those observed in diabetic ketoacidosis cases. Additionally, mean IPA values were lower than acetone values in each analyzed substrate, and the IPA/acetone blood ratio was below 1.0 in all cases. Based on the results of our investigations, we concluded that the systematic determination of IPA and ketone body levels in several biological samples should be performed in order to better quantify the extent of ketoacidosis at the moment of death. 
Erythropoietin, chromogranin A, serotonin, and S100B

Quan et al. [72] investigated erythropoietin (EPO) levels in postmortem serum from various sample sites (left and right heart and the external iliac vein) in 536 forensic autopsy cases, including 27 hypothermia fatalities. They observed a marked increased EPO value in one hypothermia case which suffered from uremia under artificial hemodialysis with medical administration of an EPO agent, as well as a mild to moderate elevation of the postmortem serum EPO levels in some cases of hypothermia in elderly subjects. According to the authors, these results may suggest that an elevated EPO level is an indication of preexisting anemia, which may have been a predisposition to death. Yoshida et al. [39] investigated chromogranin $\mathrm{A}(\mathrm{CgA})$ and catecholamine levels in postmortem serum from right heart blood and cerebrospinal fluid in 298 forensic autopsy cases. They observed that the hypothermia cases had serum $\mathrm{CgA}$ levels significantly lower and cerebrospinal fluid $\mathrm{CgA}$ levels significantly higher than cases with other causes of death. Additionally, these same hypothermia cases displayed lower serum adrenaline and noradrenaline levels and relatively high ratios of cerebrospinal fluid $\mathrm{CgA}$ to adrenaline and noradrenaline. Lastly, the cerebrospinal fluid levels of $\mathrm{CgA}$ were higher than $\mathrm{CgA}$ postmortem serum levels in most cases of hypothermia, with a moderate negative correlation between the postmortem serum and cerebrospinal fluid levels. Since hypothermia cases tended to display low hypothalamus neuronal $\mathrm{CgA}$ immunopositivity with a positive correlation to CSF CgA levels, the authors postulated that there was a terminal state of hypothalamus dysfunction involving the depletion of $\mathrm{CgA}$-containing secretory granules in prolonged death due to cold exposure. Quan et al. [73] investigated serotonin (5-HT) levels in cerebrospinal and pericardial fluids regarding the cause of death in 351 medicolegal autopsy cases. They found that the hypothermia cases tended to have low 5-HT levels in both fluids and postulated the usefulness of this marker for investigating hypothermia as the cause of death in the absence of morphological evidence. Li et al. [74] evaluated S100B levels in postmortem serum obtained from the right heart blood and cerebrospinal fluid regarding the cause of death in 216 medicolegal autopsy cases and observed lower S100B levels in the cerebrospinal fluid in hypothermia cases when compared with other causes of death.

\section{C-reactive protein and neopterin}

Fujita et al. [75] investigated C-reactive protein levels in postmortem serum obtained from the right cardiac blood of 408 medicolegal autopsy cases, including 216 cases of acute death (<30 min) and 192 cases of nonacute death (>30 min). Cold exposure cases were classified as nonacute deaths and short survivors (survival time up to $6 \mathrm{~h}$ ) and displayed moderately elevated CRP levels (between 1 and $10 \mathrm{mg} / \mathrm{l}$ ) compared with the other groups. Maeda et al. [76] analyzed urea nitrogen, creatinine, and CRP in postmortem serum from the peripheral external iliac vein (or right heart chamber when peripheral blood was not available) in 429 medicolegal autopsy cases. They observed that the hypothermia fatalities had concomitant, significant elevations in the postmortem serum levels of urea nitrogen ( $>50 \mathrm{mg} / \mathrm{dl})$, creatinine $(>2 \mathrm{mg} / \mathrm{dl})$, and CRP $(>2 \mathrm{mg} /$ dl). Ishikawa et al. [77] studied the concentrations of CRP and neopterin in postmortem serum blood from different sampling sites (subclavian and external iliac veins as well as left and right heart). The authors observed moderately elevated CRP levels $(1-10 \mathrm{mg} / \mathrm{dl})$ in fatal hypothermia cases and a moderate increase in the level of neopterin. The increased CRP levels were interpreted by the authors as the consequence of a survival period greater than 6-12 $\mathrm{h}$ with complications of frostbite and/or pneumonia. Conversely, moderate increases in neopterin levels (50-200 nmol/l) occurred in cases with survival times shorter than $24 \mathrm{~h}$ and were explained as the result of a rapid macrophage activation due to tissue injury.

\section{Conclusions}

The aim of this article was to review the medicolegal literature covering the postmortem biochemical investigations of hypothermia fatalities (Table 1). We focused on previously performed biochemical investigations and did not expand upon histological and immunohistochemical studies. This examination has disclosed some interesting aspects leading us to formulate some preliminary conclusions. Firstly, most studies correlate hypothermia fatalities with a specific biochemical marker, sometimes in combination with histological and immunohistochemical findings. However, apart from the studies performed by Ishikawa et al. on pituitary hormones, no studies have associated macroscopic and microscopic findings with a pattern of biochemical parameters (e.g., ketone bodies and hormones). In our opinion, the reasons for this approach are that postmortem biochemical investigations are largely unfamiliar, and their diagnostic potential is still greatly unrecognized. Consequently, postmortem biochemistry remains an unexplored diagnostic tool for most forensic pathologists and is not routinely performed.

Moreover, the biochemical investigations concerning specific molecules have only been pursued by a distinct group of authors. As a consequence, most of these studies represent isolated investigations that focus on specific biochemical markers whose results have never been put into question, validated, confirmed, or invalidated.

Additionally, based on this incomplete diagnostic approach, it becomes laborious to formulate an exhaustive 
Table 1 Summary of reports describing postmortem biochemical investigations and main results in hypothermia fatalities

\begin{tabular}{|c|c|c|c|}
\hline Analyte(s) & Author(s) and reference(s) & Biological fluid(s) & Main result(s) in hypothermia fatalities \\
\hline \multicolumn{4}{|l|}{ Electrolytes } \\
\hline Magnesium & Mant $[43,44]$ & $\mathrm{VH}$ & $\begin{array}{l}\text { No correlation between magnesium levels and } \\
\text { hypothermia duration }\end{array}$ \\
\hline $\begin{array}{l}\text { Calcium and } \\
\text { magnesium }\end{array}$ & Li et al. [45] & $\mathrm{PF}$ & $\begin{array}{l}\text { Higher calcium concentrations, lower magnesium } \\
\text { levels and } \mathrm{Mg} / \mathrm{Ca} \text { ratios }\end{array}$ \\
\hline Calcium and sodium & Jacubeniene et al. $[46,47]$ & Tissues & $\begin{array}{l}\text { No significant differences in the calcium levels, higher } \\
\text { sodium levels in skeletal muscle tissue }\end{array}$ \\
\hline \multirow[t]{7}{*}{ Catecholamines } & Hirvonen [48] & Urine & $\begin{array}{l}\text { Histamine and serotonin could not be regarded as reliable } \\
\text { tests for hypothermia }\end{array}$ \\
\hline & & & $\begin{array}{l}\text { Higher catecholamine concentrations in hypothermia } \\
\text { fatalities }\end{array}$ \\
\hline & $\begin{array}{l}\text { Hirvonen and Huttunen } \\
\text { [49] }\end{array}$ & Urine & $\begin{array}{l}\text { Higher adrenaline and noradrenaline } \\
\text { concentrations, adrenaline levels increased } \\
\text { proportionally more than noradrenaline }\end{array}$ \\
\hline & Sadler and Pounder [53] & Urine & $\begin{array}{l}\text { Urine catecholamine levels were related to urine } \\
\text { creatinine excretion }\end{array}$ \\
\hline & Zhu et al. [54] & $\begin{array}{l}\text { Postmortem serum from } \\
\text { different sampling sites }\end{array}$ & Low catecholamine levels \\
\hline & Ishikawa et al. [38] & $\mathrm{CSF}$ & $\begin{array}{l}\text { Positive correlation between noradrenaline levels } \\
\text { and noradrenaline immunopositivity in the } \\
\text { adrenal medulla }\end{array}$ \\
\hline & Pakanen et al. [6] & Urine & $\begin{array}{l}\text { Increased adrenaline levels, adrenaline-to- } \\
\text { noradrenaline ratio as the best indicator }\end{array}$ \\
\hline \multirow[t]{3}{*}{ Glucose } & Hirvonen [48] & Urine & $\begin{array}{l}\text { Test for urinary glucose positive in some hypothermia } \\
\text { case }\end{array}$ \\
\hline & Bray et al. $[55,56]$ & $\mathrm{VH}$ & Higher vitreous glucose concentrations \\
\hline & Coe $[57]$ & $\mathrm{VH}$ & Significant mean elevation of vitreous glucose levels \\
\hline \multirow{3}{*}{$\begin{array}{l}\text { Urea nitrogen, } \\
\text { creatinine, } \\
\text { uric acid }\end{array}$} & Zhu et al. [58] & $\begin{array}{l}\text { Postmortem serum from } \\
\text { different sampling sites }\end{array}$ & Urea nitrogen elevation in heart blood postmortem serum \\
\hline & Zhu et al. [59] & $\mathrm{PF}$ & $\begin{array}{l}\text { Mild to moderate elevations in urea nitrogen, } \\
\text { creatinine, and uric acid levels }\end{array}$ \\
\hline & Zhu et al. [60] & Postmortem serum and PF & Increased urea nitrogen and uric acid in both fluids \\
\hline Ketone bodies & $\begin{array}{l}\text { Teresiński et al. [61-63], } \\
\text { Palmiere [64] }\end{array}$ & Blood, urine, $\mathrm{VH}$ & Increased ketone levels in free-ethanol hypothermia cases \\
\hline \multicolumn{4}{|c|}{ Markers of cardiac function } \\
\hline $\mathrm{cTnT}$ & Zhu et al. [65] & $\begin{array}{l}\text { PF and postmortem serum } \\
\text { from different sampling } \\
\text { sites }\end{array}$ & $\mathrm{cTnT}$ at levels lower than the cutoff values \\
\hline ANP BNP & Zhu et al. [67] & $\mathrm{PF}$ & $\begin{array}{l}\text { Increased levels (contribution of cardiac failure in } \\
\text { the death process) }\end{array}$ \\
\hline $\begin{array}{l}\text { Myoglobin, cTnI, } \\
\text { CK-MB }\end{array}$ & Wang et al. [69] & $\begin{array}{l}\text { Right heart blood } \\
\text { postmortem } \\
\text { serum, PF, and CSF }\end{array}$ & $\begin{array}{l}\text { Lower CK-MB levels in CSF and serum } \\
\text { Lower cTnI and myoglobin in all substrates } \\
\text { Mild CK-MB elevation in PF }\end{array}$ \\
\hline \multicolumn{4}{|l|}{ Pituitary hormones } \\
\hline $\mathrm{ACTH}$ & Ishikawa et al. [36] & $\begin{array}{l}\text { Right heart blood } \\
\text { postmortem } \\
\text { serum and CSF }\end{array}$ & $\begin{array}{l}\text { ACTH serum levels similar to reference value } \\
\text { ACTH levels in CSF significantly lower when compared } \\
\text { to the other study groups }\end{array}$ \\
\hline TSH & Ishikawa et al. [37] & $\begin{array}{l}\text { Right heart blood } \\
\text { postmortem } \\
\text { serum and CSF }\end{array}$ & Lower serum and CSF levels than the other study groups \\
\hline $\mathrm{GH}$ & Ishikawa et al. [70] & $\begin{array}{l}\text { Right heart blood } \\
\text { postmortem serum }\end{array}$ & $\begin{array}{l}\text { Elevated serum levels when compared with } \\
\text { clinical reference ranges }\end{array}$ \\
\hline \multirow[t]{2}{*}{ Isopropyl alcohol } & Teresiński et al. [63] & Blood & Increased levels \\
\hline & Palmiere et al. [71] & Blood, urine, vitreous & Increased levels \\
\hline Erythropoietin & Quan et al. [72] & & \\
\hline
\end{tabular}


Table 1 (continued)

\begin{tabular}{|c|c|c|c|}
\hline Analyte(s) & Author(s) and reference(s) & Biological fluid(s) & Main result(s) in hypothermia fatalities \\
\hline & & $\begin{array}{l}\text { Postmortem serum } \\
\text { from different } \\
\text { sampling sites }\end{array}$ & $\begin{array}{l}\text { Elevated EPO levels can indicate preexisting } \\
\text { anemia }\end{array}$ \\
\hline \multirow{3}{*}{$\begin{array}{l}\mathrm{CgA} \text { and } \\
\text { catecholamines }\end{array}$} & \multirow[t]{3}{*}{ Yoshida et al. [39] } & \multirow{3}{*}{$\begin{array}{l}\text { Right heart blood } \\
\text { postmortem serum } \\
\text { and CSF }\end{array}$} & Lower serum and higher CSF CgA levels \\
\hline & & & Lower serum adrenaline and noradrenaline levels \\
\hline & & & CSF CgA levels higher then serum levels \\
\hline Serotonin & Quan et al. [73] & CSF and PF & Low serotonin levels in both fluids \\
\hline S100B & Li et al. [74] & $\begin{array}{l}\text { Right heart blood } \\
\text { postmortem serum } \\
\text { and CSF }\end{array}$ & Lower S100B levels in CSF \\
\hline CRP & Fujita et al. [75] & $\begin{array}{l}\text { Right heart blood } \\
\text { postmortem serum }\end{array}$ & Moderately elevated CRP levels \\
\hline $\begin{array}{l}\mathrm{CRP} \text {, urea nitrogen, and } \\
\text { creatinine }\end{array}$ & Maeda et al. [76] & Postmortem serum & $\begin{array}{l}\text { Increased urea nitrogen, creatinine, and CRP } \\
\text { levels }\end{array}$ \\
\hline CRP and neopterin & Ishikawa et al. [77] & $\begin{array}{l}\text { Postmortem serum from } \\
\text { different sampling sites }\end{array}$ & $\begin{array}{l}\text { Moderately elevated CRP (survival time }>6-12 \mathrm{~h} \text { ) } \\
\text { and neopterin (survival time }<24 \mathrm{~h} \text { ) levels }\end{array}$ \\
\hline
\end{tabular}

$V H$ vitreous humor, $P F$ pericardial fluid, $C S F$ cerebrospinal fluid, $A C T H$ adrenocorticotropic hormone, $G H$ growth hormone, $c T n T$ cardiac troponin $\mathrm{T}, c T n I$ cardiac troponin I, $A N P$ atrial natriuretic peptide, $B N P$ brain natriuretic peptide, $C K-M B$ creatine kinase $\mathrm{MB}, C g A$ chromogranin A, $C R P$ Creactive protein

hypothesis on the physiopathology of hypothermia in its entirety. Indeed, the biochemical changes that characterize hypothermia have been examined in a fragmented fashion as if they were individual, uncorrelated metabolic pathways and not different aspects of the same metabolic phenomenon.

Lastly, the increased or decreased levels of some of the analyzed markers that have been observed in cases of hypothermia cannot be unambiguously explained. The increased levels of certain molecules are simply the consequence of nonspecific changes (e.g., hypoxia and cardiac failure) and cannot be specifically related to hypothermia. Other markers are due to the expression of preexisting diseases, and their changes may provide information to better understand individual cases. Therefore, increased or decreased levels of a particular marker may not be precisely interpreted, and the usefulness of such markers in the understanding of hypothermia physiopathology remains indeterminate.

In our opinion, a parallel investigation pertaining to several biochemical markers in a group of hypothermia cases compared with a group of control cases would allow currently published results related to specific molecules to be validated and permit a more integrated investigation into the metabolic and biochemical changes that occur during hypothermia.

\section{References}

1. Türk EE (2010) Hypothermia. Forensic Sci Med Pathol 6:106-115

2. Saukko P, Knight B (2004) Neglect, starvation and hypothermia. In: Knight's forensic pathology. 3rd Ed. Arnold Publishers, London, pp 414-18
3. Taylor AJ, McGwin G Jr, Davis GG, Brissie RM, Holley TD, Rue LW 3rd (2001) Hypothermia deaths in Jefferson County, Alabama. Inj Prev 7:141-145

4. Lim C, Duflou J (2008) Hypothermia fatalities in a temperate climate: Sydney, Australia. Pathology 40:46-51

5. Dolinak D, Matshes E, Lew E (2005) Hypothermia. In: Forensic pathology: principles and practice. Elsevier, San Diego, pp 248-9.

6. Pakanen L, Kortelainen ML, Särkioja T, Porvari K (2011) Increased adrenaline to noradrenaline ratio is a superior indicator of antemortem hypothermia compared with separate catecholamine concentrations. J Forensic Sci 56:1213-1218

7. Preuss J, Dettmeyer R, Poster S, Lignitz E, Madea B (2008) The expression of heat shock protein 70 in kidneys in cases of death due to hypothermia. Forensic Sci Int 176:248-252

8. Hejna P, Zátopková L, Tsokos M (2012) The diagnostic value of synovial membrane hemorrhage and bloody discoloration of synovial fluid ("inner knee sign") in autopsy cases of fatal hypothermia. Int J Legal Med 126:415-419

9. Nikolić S, Zivković M, Zivković V, Juković F (2010) Hypothermia as the cause of death in forensic pathology: autopsy study. Srp Arh Celok Lek 138:467-472

10. Madea B, Tsokos M, Preuss J (2008) Death due to hypothermiamorphological findings, their pathogenesis and diagnostic value. In: Tsokos M (ed) Forensic pathology reviews, vol 5. Humana Press, Totowa, pp 3-21

11. Ogata M, Ago K, Kondo T, Kasai K, Ishikawa T, Mikuzami H (2007) A fatal case of hypothermia associated with hemorrhages of the pectoralis minor, intercostals, and iliopsoas muscles. Am J Forensic Med Pathol 28:348-352

12. Nixdorf-Miller A, Hunsaker DM, Hunsaker JC 3rd (2006) Hypothermia and hyperthermia medicolegal investigation of morbidity and mortality from exposure to environmental temperature extremes. Arch Pathol Lab Med 130:1297-1304

13. Hottmar P, Hejna P (2005) Death due to fatal hypothermia in victims dissected in Department of Forensic Medicine in Hradec Králové between 1992-2003. Soud Lek 50:38-41

14. Madea B, Preuss J, Henn V, Lignitz E (2004) Morphological findings in fatal hypothermia and their pathogenesis. In: Oehmichen M (ed) Hypothermia-clinical, pathomorphological 
and forensic features. Schmidt-Römhild, Lübeck, pp 181204

15. Hirvonen J (2000) Some aspects on death in the cold and concomitant frostbites. Int J Circumpolar Health 59:131-136

16. Pliushcheva TV, Alisievich VI (2000) Pathogenesis of Vishnevsky's spots in death from excessive cooling. Sud Med Ekspert 43:11-14

17. Wolf DA, Aronson JF, Rajaraman S, Veasey SP 3rd (1999) Wischnewski ulcers and acute pancreatitis in two hospitalized patients with cirrhosis, portal vein thrombosis, and hypothermia. J Forensic Sci 44:1082-1085

18. Mizukami H, Shimizu K, Shiono H, Uezono T, Sasaki M (1999) Forensic diagnosis of death from cold. Leg Med (Tokyo) 1:204-209

19. Ward ME, Cowley AR (1999) Hypothermia: a natural cause of death. Am J Forensic Med Pathol 20:383-386

20. Tributsch W, Ambach E, Henn R (1992) Forensic medicine aspects of death caused by hypothermia in high altitude. Beitr Gerichtl Med 50:337-341

21. Sigrist T, Markwalder C, Dirnhofer R (1990) Changes in skeletal muscles in death caused by hypothermia. Z Rechsmed 103:463-472

22. Birchmeyer MS, Mitchell EK (1989) Wischnewski revisited. The diagnostic value of gastric mucosal ulcers in hypothermic deaths. Am J Forensic Med Pathol 10:28-30

23. Schneider V, Klug E (1980) Death by hypothermia. Are there any new diagnostic aspects? Z Rechtsmed 86:59-69

24. Tsokos M, Rothschild MA, Madea B, Risse M, Sperhake JP (2006) Histological and immunohistochemical study of Wischnewsky spots in fatal hypothermia. Am J Forensic Med Pathol 27:70-74

25. Türk EE, Sperhake JP, Madea B, Preuss J, Tsokos M (2006) Immunohistochemical detection of hemoglobin in frost erythema. Forensic Sci Int 158:131-134

26. Preuss J, Thierauf A, Dettmeyer R, Madea B (2007) Wischnewsky's spots in an ectopic stomach. Forensic Sci Int 169:220-222

27. Aghayev E, Thali MJ, Jackowski C, Sonnenschein M, Dirnhofer R, Yen K (2008) MRI detects hemorrhages in the muscles of the back in hypothermia. Forensic Sci Int 176:183-186

28. Preuss J, Dettmeyer R, Lignitz E, Madea B (2004) Fatty degeneration in renal tubule epithelium in accidental hypothermia victims. Forensic Sci Int 141:131-135

29. Byard RW, Zhou C (2010) Erosive gastritis, Armanni-Ebstein phenomenon and diabetic ketoacidosis. Forensic Sci Med Pathol 6:304-306

30. Zhou C, Byard RW (2011) Armanni-Ebstein phenomenon and hypothermia. Forensic Sci Int 206:82-84

31. Hirvonen J, Penttinen J, Huttunen P, Saukko P (1980) Changes in the myocardium and skeletal muscle in guinea pigs in cold exposure with and without ethanol. Z Rechsmed 84:195-207

32. Preuss J, Dettmeyer R, Lignitz E, Madea B (2006) Fatty degeneration of myocardial cells as a sign of death due to hypothermia versus degenerative deposition of lipofuscin. Forensic Sci Int $159: 1-5$

33. Preuss J, Lignitz E, Dettmeyer R, Madea B (2007) Pancreatic changes in cases of death due to hypothermia. Forensic Sci Int 166:194-198

34. Ishikawa T, Miyaishi S, Tachibana T, Ishizu H, Zhu BL, Maeda $\mathrm{H}$ (2004) Fatal hypothermia related vacuolation of hormoneproducing cells in the anterior pituitary. Leg Med (Tokyo) 6:157163

35. Doberentz E, Preuss-Wössner J, Kuchelmeister K, Madea B (2011) Histological examination of the pituitary glands in cases of fatal hypothermia. Forensic Sci Int 207:46-49

36. Ishikawa T, Quan L, Li DR, Zhao D, Michiue T, Hamel M, Maeda $\mathrm{H}$ (2008) Postmortem biochemistry and immunohistochemistry of adrenocorticotropic hormone with special regard to fatal hypothermia. Forensic Sci Int 179:147-151
37. Ishikawa T, Michiue T, Zhao D, Komatsu A, Azuma Y, Quan L, Hamel M, Maeda H (2009) Evaluation of postmortem serum and cerebrospinal fluid levels of thyroid-stimulating hormone with special regard to fatal hypothermia. Leg Med (Tokyo) 11:S228$\mathrm{S} 230$

38. Ishikawa T, Yoshida C, Michiue T, Perdekamp MG, Pollak S, Maeda $H$ (2010) Immunohistochemistry of catecholamine in the hypothalamic-pituitary-adrenal system with special regard to fatal hypothermia and hyperthermia. Leg Med (Tokyo) 12:121-127

39. Yoshida C, Ishikawa T, Michiue T, Quan L, Maeda H (2011) Postmortem biochemistry and immunohistochemistry of chromogranin $\mathrm{A}$ as a stress marker with special regard to fatal hypothermia and hyperthermia. Int J Legal Med 125:11-20

40. Quan L, Ishikawa T, Michiue T, Li DR, Zhao D, Zhu BL, Maeda H (2005) Quantitative analysis of ubiquitin-immunoreactivity in the midbrain periaqueductal gray matter with regard to the cause of death in forensic autopsy. Leg Med (Tokyo) 7:151-156

41. Ishikawa T, Zhu BL, Li DR, Zhao D, Michiue T, Maeda H (2007) Immunohistochemical investigation of ubiquitin and myoglobin in the kidney in medicolegal autopsy cases. Forensic Sci Int 171:136-141

42. Kita T (2000) The role of heat shock proteins on the disordered tissues: implication for the pathogenesis and diagnostics in the forensic practice. Nihon Hoigaku Zasshi 54:367-371

43. Mant AK (1964) Some post-mortem observation in accidental hypothermia. Med Sci Law 4:44-46

44. Mant AK (1969) Autopsy diagnosis of accidental hypothermia. J Forensic Med 16:126-129

45. Li DR, Quan L, Zhu BL, Ishikawa T, Michiue T, Zhao D, Yoshida C, Chen JH, Wang Q, Komatsu A, Azuma Y, Maeda H (2009) Evaluation of postmortem calcium and magnesium levels in the pericardial fluid with regard to the cause of death in medicolegal autopsy. Leg Med (Tokyo) 11:S276-S278

46. Jakubeniene M, Chaker GA, Becelis A, Malakiene D, Raudys R (2009) Investigation of calcium and sodium in postmortem material as biochemical markers defining the cause of death from hypothermia. Leg Med (Tokyo) 11:S304-S306

47. Jakubeniene M, Irnius A, Chaker GA, Paliulis JM, Bechelis A (2009) Post-mortem investigation of calcium content in liver, heart, and skeletal muscle in accidental hypothermia cases. Forensic Sci Int 190:87-90

48. Hirvonen J (1976) Necropsy findings in fatal hypothermia cases. Forensic Sci 8:155-164

49. Hirvonen J, Huttunen P (1982) Increased urinary concentration of catecholamines in hypothermia deaths. J Forensic Sci 27:264-271

50. Hirvonen J, Huttunen P, Nuutinen L, Pekkariken A (1978) Cathecolamines and free fatty acids in plasma of patients undergoing cardiac operation with hypothermia bypass. J Clin Pathol 31:949955

51. Hanhela R, Hollmen A, Huttunen P, Hirvonen J (1990) Plasma catecholamines, corticosterone, glucose and fatty acids concentrations and mean arterial pressure and body temperature in haemorrhagic hypovolaemia, hypothermia and a combination of these in the rabbit. Acta Physiol Scand 139:441-449

52. Hirvonen J, Huttunen P (1995) Hypothermia markers: serum, urine and adrenal gland catecholamines in hypothermic rats given ethanol. Forensic Sci Int 72:125-133

53. Sadler DW, Pounder DJ (1995) Urinary catecholamines as markers of hypothermia. Forensic Sci Int 76:227-230

54. Zhu BL, Ishikawa T, Michiue T, Li DR, Zhao D, Quan L, Oritani S, Bessho Y, Maeda H (2007) Postmortem serum catecholamine levels in relation to the cause of death. Forensic Sci Int 173:122-129

55. Bray M, Luke JL, Blackbourne BD (1983) Vitreous humor chemistry in deaths associated with rapid chilling and prolonged freshwater immersion. J Forensci Sci 28:588-593

56. Bray M (1984) The eye as a chemical indicator of environmental temperature at the time of death. J Forensic Sci 29:396-403 
57. Coe JI (1984) Hypothermia: autopsy findings and vitreous glucose. J Forensic Sci 29:389-395

58. Zhu BL, Ishida K, Quan L, Taniguchi M, Oritani S, Li DR, Fujita MQ, Maeda H (2002) Postmortem serum uric acid and creatinine levels in relation to the causes of death. Forensic Sci Int 125:59-66

59. Zhu BL, Ishikawa T, Michiue T, Li DR, Zhao D, Quan L, Maeda H (2005) Evaluation of postmortem urea nitrogen, creatinine and uric acid levels in pericardial fluid in forensic autopsy. Leg Med (Tokyo) 7:287-292

60. Zhu BL, Ishikawa T, Michiue T, Tanaka S, Zhao D, Li DR, Quan L, Oritani S, Maeda H (2007) Differences in postmortem urea nitrogen, creatinine and uric acid levels between blood and pericardial fluid in acute death. Leg Med (Tokyo) 9:115-122

61. Teresiński G, Buszewicz G, Madro R (2002) The influence of ethanol on the level of ketone bodies in hypothermia. Forensic Sci Int 127:88-96

62. Teresiński G, Buszewicz G, Madro R (2005) Biochemical background of ethanol-induced cold susceptibility. Leg Med (Tokyo) 7:15-23

63. Teresiński G, Buszewicz G, Madro R (2009) Acetonaemia as an initial criterion of evaluation of a probable cause of sudden death. Leg Med (Tokyo) 11:18-24

64. Palmiere C, Mangin P (2012) Postmortem chemistry update part I. Int J Legal Med 126:187-198

65. Zhu BL, Ishikawa T, Michiue T, Li DR, Zhao D, Oritani S, Kamikodai Y, Tsuda K, Okazaki S, Maeda H (2006) Postmortem cardiac troponin T levels in the blood and pericardial fluid. Part 1. Analysis with special regard to traumatic causes of death. Leg Med (Tokyo) 8:86-93

66. Zhu BL, Ishikawa T, Michiue T, Li DR, Zhao D, Kamikodai Y, Tsuda K, Okazaki S, Maeda H (2006) Postmortem cardiac tropo$\operatorname{nin} \mathrm{T}$ levels in the blood and pericardial fluid. Part 2: analysis for application in the diagnosis of sudden cardiac death with regard to pathology. Leg Med (Tokyo) 8:94-101

67. Zhu BL, Ishikawa T, Michiue T, Li DR, Zhao D, Tanaka S, Kamikodai Y, Tsuda K, Okazaki S, Maeda H (2007) Postmortem pericardial natriuretic peptides as markers of cardiac function in medico-legal autopsies. Int J Legal Med 121:28-35

68. Zhu BL, Ishikawa T, Michiue T, Li DR, Zhao D, Bessho Y, Kamikodai Y, Tsuda K, Okazaki S, Maeda H (2007) Postmortem cardiac troponin I and creatine kinase MB levels in the blood and pericardial fluid as markers of myocardial damage in medicolegal autopsy. Leg Med (Tokyo) 9:241-250

69. Wang Q, Michiue T, Ishikawa T, Zhu BL, Maeda H (2011) Combined analyses of creatine kinase $\mathrm{MB}$, cardiac troponin I and myoglobin in pericardial and cerebrospinal fluids to investigate myocardial and skeletal muscle injury in medico-legal autopsy cases. Leg Med (Tokyo) 13:226-232

70. Ishikawa T, Michiue T, Maeda H (2011) Evaluation of postmortem serum and cerebrospinal fluid growth hormone levels in relation to the cause of death in forensic autopsy. Hum Cell 24:74-77

71. Palmiere C, Sporkert F, Werner D, Bardy D, Augsburger M, Mangin P (2012) Blood, urine and vitreous isopropyl alcohol as biochemical markers in forensic investigations. Leg Med (Tokyo) 14:17-20

72. Quan L, Zhu BL, Ishikawa T, Michiue T, Zhao D, Ogawa M, Maeda H (2010) Postmortem serum erythropoietin level as a marker of survival time in injury deaths. Forensic Sci Int 200:117-122

73. Quan L, Ishikawa T, Hara J, Michiue T, Chen JH, Wang Q, Zhu BL, Maeda H (2011) Postmortem serotonin levels in cerebrospinal and pericardial fluids with regard to the cause of death in medicolegal autopsy. Leg Med (Tokyo) 13:75-78

74. Li DR, Michiue T, Zhu BL, Ishikawa T, Quan L, Zhao D, Yoshida C, Chen JH, Wang Q, Komatsu A, Azuma Y, Maeda H (2009) Evaluation of postmortem S100B levels in the cerebrospinal fluid with regard to the cause of death in medicolegal autopsy. Leg Med (Tokyo) 11:S273-S275

75. Fujita MQ, Zhu BL, Ishida K, Quan L, Oritani S, Maeda H (2002) Serum C-reactive protein levels in postmortem blood - an analysis with special reference to the cause of death and survival time. Forensic Sci Int 130:160-166

76. Maeda H, Zhu BL, Bessho Y, Ishikawa T, Quan L, Michiue T, Zhao D, Li DR, Komatsu A (2008) Postmortem serum nitrogen compounds and C-reactive protein levels with special regard to investigation of fatal hyperthermia. Forensic Sci Med Pathol 4:175-180

77. Ishikawa T, Hamel M, Zhu BL, Li DR, Zhao D, Michiue T, Maeda H (2008) Comparative evaluation of postmortem serum concentrations of neopterin and C-reactive protein. Forensic Sci Int 179:135-143 